\title{
Temporal experience as a function of organization in memory
}

\author{
ROBERT M. MULLIGAN and H. R. SCHIFFMAN \\ Rutgers University, Busch Campus, New Brunswick, New Jersey 08903
}

\begin{abstract}
The relationship between memory organization and perceived duration was investigated in two experiments. Degree of organization of the memory representation for an interval was assumed to be inversely related to the complexity of the interval's content. Organization was manipulated by presenting an ambiguous stimulus during the interval either with or without the aid of a code that simplified the stimulus. Subjects judged the duration of a 60-sec interval by the method of magnitude estimation and then recalled the interval's contents. In both experiments, facilitating memory organization by presenting the simplifying code before the interval was found to shorten apparent duration. In the second experiment, presenting the code after the stimulus interval also significantly shortened apparent duration when compared with the "no-code" condition. In general, the results were consistent with Ornstein's (1969) storage size notion of the experience of duration. Some limitations of the storage size hypothesis are discussed.
\end{abstract}

Ornstein (1969) proposed that the perception of a temporal interval is affected by specifiable properties of the input stimuli perceived within the interval (e.g., number of items), as these properties serve to determine the encoding of perceived stimuli and their subsequent storage in memory. A basic premise of the metaphor is that the amount of data stored in memory and, more importantly, the manner in which it is encoded for storage determine the duration experience such that apparent duration lengthens as "storage size" increases. It follows, Ornstein argued, that manipulation of the stored representation of the interval after the input stage-the reorganization of memory storage-should predictably alter the perception of temporal experience.

There is clear evidence that the amount of stimulus information in an interval is positively correlated with perceived duration (e.g., Fraisse, 1963; Ornstein, 1969; Schiffman \& Bobko, 1977); however, the role of the organization of an interval's contents and its corresponding memory representation has not received much study. Ornstein (Experiment 8) has offered some evidence that the degree of organization of an interval's memory representation affects apparent duration. He attempted to manipulate the degree of organization of memory storage by varying the complexity of the stimulus items presented during the interval. When presented with a complex stimulus (ambiguous line drawing) for $60 \mathrm{sec}$, subjects given a simplifying code (the "coded set") for the stimulus reported shorter apparent durations than did subjects unaided by the code. These results obtained

This research was supported by funds from the Research Council of Rutgers University Grant 07-2109 and the Busch Memorial Fund. Requests for reprints should be sent to H. R. Schiffman, Department of Psychology, Rutgers University, Busch Campus, New Brunswick, New Jersey 08903. whether the code was given before or after the 60 -sec stimulus presentation.

In line with his storage size hypothesis, Ornstein (1969) argued that, in the absence of the simplifying code, the ambiguous drawing constituted a more complex stimulus and therefore required more storage space than it did when the code was present. The implication of these findings is that organization of the stored representation of the stimuli perceived within the interval affects apparent duration such that more organized (less complex) intervals are subjectively shorter than intervals during which the same stimuli are presented in a less organized manner.

Although Ornstein's (1969) results are often cited and the "storage size" hypothesis is accepted as a reasonable explanation for the perception of duration in retrospect, little additional evidence for the hypothesis has been reported. The present investigation was designed to confirm and extend Ornstein's storage size notion.

\section{EXPERIMENT 1}

Experiment 1 attempted to replicate the findings of Ornstein's (1969) Experiment 8 using slightly different stimulus materials and procedures. Ornstein's subjects compared the interval during which they viewed the test figure with a standard interval during which they heard a recording of random sounds. In the present experiment, both test and standard intervals were visually defined.

Second, the simplifying code that rendered the test figure more readily interpretable in the present study was in the form of a verbal label or caption and was the same for both "code-before" (CB) and "code-after" (CA) conditions. In his CB condition, Ornstein (1969) used a visual cue (the "coded set") to provide subjects the appropriate interpretation of the test figure; for the CA 
group, he provided both the visual cue and a verbal cue, a sentence divulging the appropriate interpretation.

These minor changes in method do not delimit the predictions made by the storage size hypothesis, but they should extend its generality. Subjects given the figure's caption should encode the interval more efficiently and thus judge it as shorter in duration than would subjects not given the simplifying code.

\section{Method}

Materials and Design. The test stimulus was a "droodle": a simple line drawing, ambiguous and uninterpretable by itself, which is disambiguated by a humorous caption (see Price, 1972). The droodle used is shown in Figure 1 with an irregular polygonal figure that served as a standard stimulus for the duration estimation procedure. The materials were presented in booklets on pages backed with black construction paper to prevent subjects from seeing previous or subsequent pages.

As in Ornstein's (1969) investigation, subjects were required to make duration judgments in one of three conditions, corresponding to the three levels of the coding variable. In the CB condition, subjects read the humorous figure caption ("an early bird who caught a very strong worm") before viewing the test figure. In the CA condition, subjects read the figure caption after viewing the test figure. The no-code (NC) group was not given the figure caption.

Procedure. The subjects, 45 volunteers from an introductory psychology course, were tested in groups of 4-8. They were randomly assigned to one of the code conditions as they arrived and were seated at tables with folders containing the experimental materials in front of them. The following directions, which the experimenter read aloud to subjects, were typed on the first page of the booklets:

"The purpose of this research is to find out something about how people remember simple line drawings. After reading these directions you will be shown two simple figures. Please study the figures carefully, as you will be asked to remember and compare them later.

"During the experiment please be careful to turn only one page at a time and only when you have been instructed to do so. Do not look ahead in the booklet or back to any previous pages. Remember to pay close attention to the figures."

Subjects then turned to the second page and viewed the standard figure for $60 \mathrm{sec}$, after which they were instructed to turn to the next page in the booklet. For subjects in the $C B$ condition, the simplifying code for the test figure was typed on this page. Subjects in the other two conditions saw the instruction, "Please wait until you are told to turn to the next page."

After a 10 -sec interval, subjects turned to the next sheet and viewed the test figure for $60 \mathrm{sec}$. Following this, the CA group was given the caption and the other two groups read the "please

a

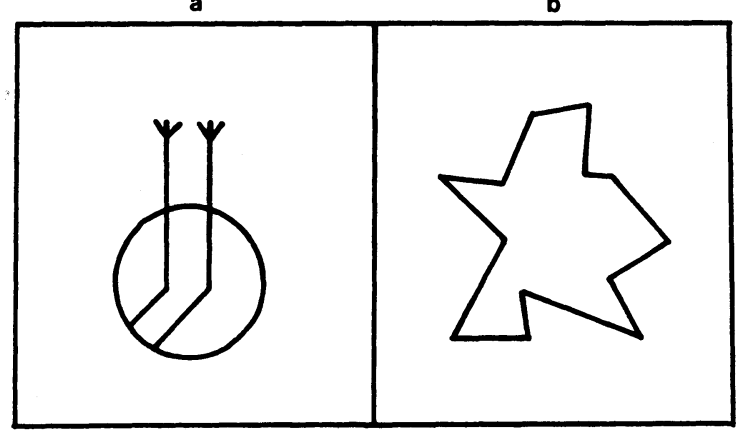

Figure 1. Figures of Experiment 1. (a) Droodle, "An early bird who caught a very strong worm," viewed during the test interval. (b) Irregular polygon viewed during the standard interval. wait ..." instruction. Ten seconds later, all subjects went on to the duration estimation task. The boundaries of each interval were marked by the experimenter's request, "Please turn to the next page in your booklet," which was cued by the display of a digital timer.

Subjects judged amount of time spent viewing the droodle by a scaling technique wherein the interval during which subjects viewed the polygon was used as the standard. The subjects were unaware until this point that they would have to make a duration judgment; that is, "temporal motive" (Doob, 1971) was aroused subsequent to the test interval. They then read the following directions: "I would now like you to compare the length of time you looked at the second figure with the time spent looking at the first figure. To do this, imagine that the top horizontal line shown below represents the length of the interval during which you looked at the first figure. Indicate how long the second interval (during which you looked at the second figure) seemed to you relative to the first interval by marking off a line segment on the lower line. Do this by making a small vertical slash across the line. If you felt that the second interval was longer than the first, mark off a line segment on the bottom line which is proportionally longer than the top line. If you felt the second interval was shorter than the first, mark off a segment shorter than the top line."

A 5-cm line segment was drawn on the page to represent the standard interval. Immediately beneath it was a $10-\mathrm{cm}$ line on which the subjects were to mark their duration estimates.

Two further tasks were included. The first asked subjects to write a description of the test figure as if they were describing it to a friend. They were told to make their descriptions accurate enough such that the other person "gets the picture." This procedure was used by Ornstein (1969) to provide "a measure of the size of the [memory] storage of that interval" (p. 92).

The final task required subjects to sketch the two experimental figures accurately but without being overly concerned with artistic detail. Subjects were also asked if they had seen the droodle before (none had), and the CA and NC groups were asked if they had been able to guess the code while examining the figure (none had).

\section{Results and Discussion}

The ratio of the line length judged to represent the duration of the test interval to the line length representing the duration of the standard interval was calculated for each subject. The effect of the coding factor on these duration judgment ratios was examined by a one-way ANOVA for randomized groups. The variable was found to have a significant effect on duration estimates $[F(2,42)=3.80, p<.05]$. The mean duration estimation ratios for each of the three groups are presented in Table 1. A Newman-Keuls multiple-comparison test showed that the test interval was judged to be significantly shorter in the $\mathrm{CB}$ condition than in the $\mathrm{NC}$ condition $(\mathrm{p}<.05)$. The mean of the $\mathrm{CA}$ group fell between those of Groups CB and NC but was not significantly different from either one. (A pilot study employing a different droodle yielded the same pattern of results.)

Although somewhat less pronounced, this result is quite similar to that reported by Ornstein (1969). When a subject is given an efficient means of encoding a simple, visually presented figure at the input stage, the interval during which the figure is viewed is judged to be shorter than when no code is provided. Also, if the appropriate code is made available immediately after the figure is viewed, the test interval tends to be judged shorter 
Table 1

Means and Standard Deviations for Duration and Verbal Description Measures in Experiment 1

\begin{tabular}{cccccc}
\hline & \multicolumn{2}{c}{$\begin{array}{c}\text { Duration Ratio } \\
\text { (Test:Standard) }\end{array}$} & & \multicolumn{2}{c}{$\begin{array}{c}\text { Number of Words in } \\
\text { Verbal Description }\end{array}$} \\
\cline { 2 - 3 } \cline { 5 - 6 } Group & Mean & SD & & Mean & SD \\
\hline CB & .69 & .17 & & 76.3 & 21.9 \\
CA & .82 & .28 & & 87.7 & 23.8 \\
NC & .90 & .19 & & 90.3 & 21.0 \\
\hline
\end{tabular}

than if no code is provided. The latter case appears to reflect the ability of some subjects to recode the memory representation of the figure into a more efficient form. That not all subjects used the new, more efficient code is suggested by the fact that the variability of the CA group duration estimates was nearly twice that of the other two groups (see Table 1). Thus, although these results do not exactly replicate Ornstein's, they can readily be accounted for by a general form of the storage size hypothesis.

Unlike Ornstein's (1969) results, however, no significant differences were observed in the present study in the number of words used to describe the droodle in the $\mathrm{CB}, \mathrm{CA}$, and NC conditions. This finding lends no support to the storage size hypothesis.

The results of the recall task indicated that subjects in all conditions had a good representation of information presented during the test interval, since all subjects were able to draw the test figure nearly perfectly. Given this result, we might conclude that differences between groups in the judged duration of the test interval were due more to differences in stimulus complexity and organization of the interval's contents in memory than to the absolute amount of information retained.

\section{EXPERIMENT 2}

The purpose of Experiment 2 was to determine whether further evidence for the storage size hypothesis could be obtained when the intervals to be judged were filled with meaningful verbal material. Experiment 2 was structurally identical to Experiment 1 except that, instead of an ambiguous line drawing, a short ambiguous paragraph provided the content for the test interval. It was expected that the duration estimation results would parallel those of Experiment 1. That is, groups given a disambiguating code should judge the duration of the test interval to be shorter than should the group not given the simplifying code.

\section{Method}

Material and Design. A 60-sec segment of tape-recorded music, played at a comfortable listening intensity (around $60 \mathrm{~dB}$ ), served as the standard stimulus interval for Experiment 2. The segment was selected from an electronic recording by Walter Carlos of Monteverdi's Orpheo Suite. The start and end of the segment were marked by a $1-\mathrm{sec} 1,000-\mathrm{Hz}$ tone. The remainder of the materials were assembled into a booklet, as in Experiment 1.

During the 60 -sec test interval subjects were given the following brief passage to read and study: "The failure is in one of four places. After determining which one it is, you must get out the equipment. Extreme caution is advisable. One of the first steps is to remove the nuts. After the exchange is made, the body is lowered and away you go."

The passage is similar to those created by Bransford and Johnson (1972) to study the role of contextual information in comprehension and recall. Sentences in the passage follow normal English construction, and the vocabulary items are used in nonmetaphorical ways; however, the passage reads ambiguously without some additional information. The requisite information for comprehension of the passage is in the form of a contextual cue that makes the passage more meaningful. The simplifying code or context for the present passage is "changing a flat tire."

The design was identical to that of Experiment 1. Preliminary testing showed that subjects were able to recall the passage nearly perfectly after the 60 -sec study period, regardless of whether they were given the simplifying code. This outcome was necessary in order for the second experiment to parallel the first, in which all subjects were able to recall the contents of the test interval.

Procedure. The procedure was similar to that used in Experiment 1 . Forty-five naive volunteers served as subjects. They first read instructions that described the music segment and paragraphs that were to follow and told subjects to "pay careful attention to both of these stimuli as you will be asked to remember and compare them later."

After receiving instructions on the use of the test booklet, the subjects heard the music segment. At the conclusion of the recording, the subjects turned to an instruction page, which, for the CB group, contained the simplifying code (i.e., "The passage you will read is about changing a flat tire"). Subjects in the other two conditions read, "Please wait until you are told to turn to the next page." After a 10-sec interval, the subjects were told to turn to the following page, on which the experimental passage was typed. The subjects studied the passage for $60 \mathrm{sec}$ and then turned to the next page, which presented the code to the CA group and the "Please wait ..." instruction to the other groups. Ten seconds later, all subjects went on to the duration estimation task. The boundaries of the 10- and 60 -sec intervals were marked by $1-\mathrm{sec} 1,000-\mathrm{Hz}$ tones on the tape.

The duration of the interval during which the passage was studied was judged as in Experiment 1. The instructions were similar to those of Experiment 1, with the music segment serving as a standard interval.

As a final task, subjects were asked to recall the passage. The instructions specified that subjects were to report the exact words if possible and, otherwise, to recall the main ideas of the sentences. The written recall protocols were scored according to the number of "idea units" (Bransford \& Johnson, 1972) correctly recalled. A fairly liberal scoring criterion was used, such that if a subject remembered the gist or main idea of each phrase or sentence, the response was scored as correct. The passage used was divided into nine idea units based on a consensus drawn by the authors from judgments made by five graduate students and faculty members.

\section{Results and Discussion}

The duration data were analyzed as they had been for Experiment 1. A one-way ANOVA showed that the administration of the code had a significant effect on judged retrospective duration $[\mathrm{F}(2,42)=4.85, \mathrm{p}<.025]$. The mean duration ratio for the $\mathrm{NC}$ group was determined by a Newman-Keuls multiple-range test to be reliably larger than the means for both the $C B$ and CA groups $(p<.05)$. As shown in Table 2 , the CB and CA means were nearly equal. The effect of the code (both in CB and CA) on judged duration of the interval during which the passage was studied was one of shortening temporal experience relative to the condition in which no code was provided. 
Table 2

Means and Standard Deviations for Duration and Recall Measures in Experiment 2

\begin{tabular}{cccccc}
\hline & \multicolumn{2}{c}{$\begin{array}{c}\text { Duration Ratio } \\
\text { (Test:Standard) }\end{array}$} & & \multicolumn{2}{c}{$\begin{array}{c}\text { Number of Idea } \\
\text { Units Recalled }\end{array}$} \\
\cline { 2 - 3 } \cline { 5 - 6 } Group & Mean & SD & & Mean & SD \\
\hline CB & .82 & .17 & & 6.9 & .16 \\
CA & .84 & .12 & & 6.1 & .19 \\
NC & .99 & .18 & & 6.5 & .16 \\
\hline
\end{tabular}

In order to parallel the first study, it was necessary that subjects in all groups in Experiment 2 have a good representation of the passage and be able to recall it equally well. A one-way ANOVA of the number of idea units recalled by each of the three groups revealed that this condition was met. There were no significant differences in the number of idea units recalled as a function of code condition $[\mathrm{F}(2,42)=.87, \mathrm{p}>.05]$. From the means in Table 2, it can be seen that, on the average, all groups recalled between six and seven of the nine idea units.

\section{GENERAL DISCUSSION}

The results of these two experiments lend support to Ornstein's (1969) storage size hypothesis. Basically, the experience of duration in retrospect (with temporal motive aroused subsequent to the interval) may be conceived of as a cognitive construction based on the representation of the interval in memory. Indeed, the results of Experiment 1 closely approximated Ornstein's (Experiment 8) results. Although judged duration in the CA group was not significantly shorter than in the NC group, the pattern of results was the same as that found by Ornstein.

Moreover, the results of Experiment 2 extend the generality of the storage size notion to include intervals filled with linguistically meaningful material. The pattern of duration judgments in this study followed exactly those in Ornstein's (1969) Experiment 8. This outcome may be interpreted, in terms of the storage size model, as follows: Although subjects in all three groups had an adequate representation of the ideas expressed in the short paragraph, CB subjects apparently encoded and stored the information in a more efficient or more organized manner. This resulted in a shortening of apparent duration when compared with the NC group. Also, subjects in the CA group had little trouble recoding the interval into a more organized form, since their duration judgments were nearly equal to those of the $C B$ group.

Although this storage size interpretation accounts for these data, some persistent problems limit its acceptability. First, in spite of instructions to study the stimuli carefully, it is impossible with the methodology employed to be certain that active study time was equal across conditions. This possibility leaves the results open to other interpretations based on differences in study time or attention. Although our observation of subjects' behavior indicated that these differences did not exist, measures should be taken in subsequent studies to assure that the effective stimuli are equated across conditions.
A second problem shared by many studies involves the presumption of an intervening variable, encoding efficiency or storage size in this case, to explain the differences in perceived durations. Some independent evidence for these hypothesized memory phenomena is necessary to strengthen the storage size argument.

Finally, it must be conceded that this support for the storage size hypothesis has been generated within a limited set of circumstances. There is some evidence that differing results can be obtained under different conditions. For example, the opposite effect of stimulus complexity and memory organization on apparent duration has been reported by Block (1974). He found that when word lists were presented in an organized fashion (blocked by categories), apparent duration of the interval during which they were perceived was reliably longer than when the words were presented in random order. Free recall and recognition measures were also significantly greater for the blocked than for the random condition.

One potentially important difference between Block's (1974) study and both Ornstein's (1969) Experiment 8 and the present experiments is the difference in the density of information presented during the test interval. In the latter experiments, subjects were given $60 \mathrm{sec}$ to study a single line drawing or a short paragraph. In Block's Experiment 2, however, a list of 80 words was presented at a rate of one four-word slide every $8 \mathrm{sec}$. This apparent difference in the demands imposed on the subject's information processing systems seems a likely cause of the differing effects of stimulus complexity and memory organization in the two types of experiments and should be examined further.

In general, the validity of the storage size hypothesis as applied to the experience of duration in retrospect remains somewhat tentative. It cannot accurately predict duration judgments in all circumstances (e.g., Block, 1974), but it can be readily modified to account for them. Indeed, Ornstein's (1969) model garners both its greatest appeal and its greatest difficulty from the fact that it is vague enough in its definition of storage size to account for many kinds of results. Yet, it continues to be a reasonable working model of duration experience in situations in which attention, motivation, temporal motive, and other variables can be controlled. The precise mechanisms involved in constructing duration experience from the stored representation of the interval, however, remain to be specified.

\section{REFERENCES}

BLock, R. A. Memory and the experience of duration retrospect. Memory \& Cognition, 1974, 2, 153-160.

Bransford, J. D., \& Johnson, M. K. Contextual prerequisites for understanding: Some investigations of comprehension and recall. Journal of Verbal Learning and Verbal Behavior, 1972, 11, 717-726.

Dоов, L. W. Patterning of time. New Haven: Yale University Press, 1971.

Fraisse, P. The psychology of time. New York: Harper \& Row, 1963.

Ornstein, R. E. On the experience of time. Middlesex, England: Penguin, 1969.

Price, R. Droodles. Los Angeles: Price/Stern/Sloan, 1972.

Schiffman, H. R., \& Bobko, D. J. The role of number and familiarity of stimuli in the perception of brief temporal intervals. American Journal of Psychology, 1977, 90, 85-93.

(Received for publication September 21, 1979.) 